
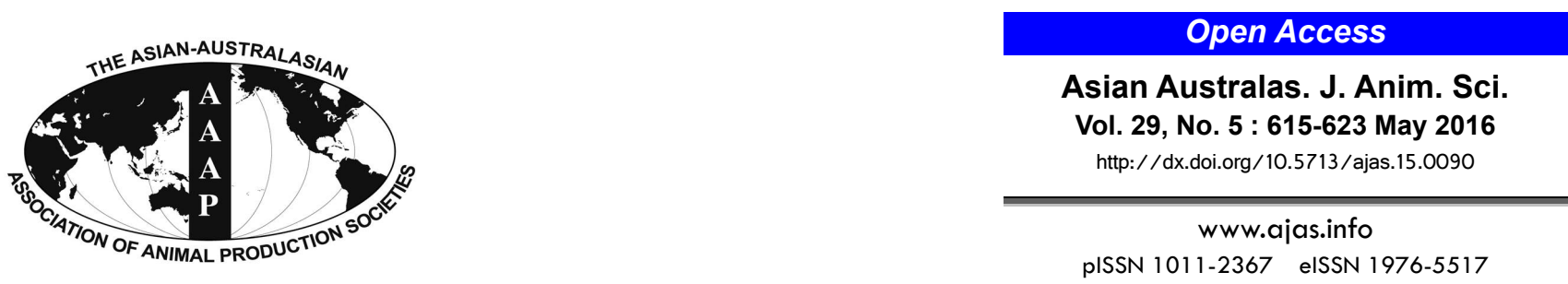

\title{
mRNA Expression of Ovine Angiopoietin-like Protein 4 Gene in Adipose Tissues
}

\author{
Jing Zhang, Jiong-Jie Jing, Xia-Li Jia, Li-Ying Qiao, Jian-Hua Liu, Chen Liang, and Wen-Zhong Liu* \\ College of Animal Science and Technology, Shanxi Agricultural University, Taigu 030801, China
}

\begin{abstract}
Angiopoietin-like protein 4 (ANGPTL4) is involved in a variety of functions, including lipoprotein metabolism and angiogenesis. To reveal the role of ANGPTL4 in fat metabolism of sheep, ovine ANGPTL4 mRNA expression was analyzed in seven adipose tissues from two breeds with distinct tail types. Forty-eight animals with the gender ratio of 1:1 for both Guangling Large Tailed (GLT) and Small Tailed Han (STH) sheep were slaughtered at 2, 4, 6, 8, 10, and 12 months of age, respectively. Adipose tissues were collected from greater and lesser omental, subcutaneous, retroperitoneal, perirenal, mesenteric, and tail fats. Ontogenetic mRNA expression of ANGPTL4 in these adipose tissues from GTL and STH was studied by quantitative real time polymerase chain reaction. The results showed that ANGPTL4 mRNA expressed in all adipose tissues studied with the highest in subcutaneous and the lowest in mesenteric fat depots. Months of age, tissue and breed are the main factors that significantly influence the mRNA expression. These results provide new insights into ovine ANGPTL4 gene expression and clues for its function mechanism. (Key Words: Adipose Tissue, ANGPTL4, mRNA Expression, Sheep)
\end{abstract}

\section{INTRODUCTION}

ANGPTL4, also known as a fasting-induced adipose factor, which is up-regulated by fasting, is one of the seven members of angiopoietin-like proteins (ANGPTLs). It is involved in angiogenesis and encoded by ANGPTL4 gene. It is secreted by a variety of tissues, mainly by liver and white adipose tissues (WAT), and exists in either native (full-length) form with molecular mass of $\sim 50 \mathrm{kDa}$ (Mandard et al., 2006) or truncated forms both in human and mouse plasma (Mandard et al., 2004; Zhu et al., 2012). Different forms can result from complex processing, including cleavage, glycosylation and oligomerization of the ANGPTL4 protein, which may be inherent to the cell line/type and species (Grootaert et al., 2012). In mice, the native form was physically associated with high density lipoprotein, whereas truncated form was associated with low density lipoprotein. In human both native and truncated forms were associated with high density lipoprotein. The

\footnotetext{
* Corresponding Author: Wen-Zhong Liu. Tel: +86-0354-6288 337, E-mail: tglwzyc@163.com

Submitted Jan. 31, 2015; Revised Apr. 6, 2015; Accepted Aug. 2, 2015
}

truncated ANGPTL4 was found to be associated with adipocyte differentiation in mice, however, only the native form was detected in adipose tissues in human (Mandard et al., 2004).

ANGPTL4 has been identified as a multifunctional signal protein. Nevertheless, growing evidence indicates that ANGPTL4 mediates the physiological fluctuations of the activity of lipoprotein lipase (LPL), an enzyme responsible for the conversion of triglycerides to monoglycerides and fatty acids from circulating lipoproteins (Dijk and Kersten., 2014). ANGPTL4 can inhibit the activity of LPL (Georgiadi et al., 2012) by disrupting enzyme dimerization to limit extracellular lipolysis. Therefore, higher ANGPTL4 expression results in elevated triglyceride (TG) in plasma (Kadomatsu et al., 2011) and thus can induce obesity, insulin resistance, hyperlipidemia etc. Besides inhibiting LPL, ANGPTL4 also potentiates the actions of catecholamines and glucocorticoids by promoting their effects on cyclic adenosine monophosphate (cAMP) dependent TG hydrolysis in mice under short and long term fasting, respectively (Gray et al., 2012). The cAMP dependent TG hydrolysis is characterized by transcriptional effects on 
many genes, including those encoding adipose triglyceride lipase, peroxisome proliferator-activated receptor alpha, peroxisome proliferator-activated receptor gamma coactivator 1-alpha, uncoupling protein-1 (UCP-1), 3hydroxyacyl-CoA dehydrogenase, diacylglycerol Oacyltransferase homolog 2, hormone sensitive lipase, perilipin, comparative gene identification-58, and ANGPTL4 itself (Mandard et al., 2006; Gray et al., 2012). ANGPTL4 can promote the intracellular lipolysis by increasing the expression of WAT genes involved in TG hydrolysis (Mandard et al., 2006). As a result, ANGPTL4 may regulate both extra- and intracellular lipolysis (Gray et al., 2012).

ANGPTL4 mRNA is expressed in many organs and tissues, highly in WAT, placenta, liver, muscle (Feingold et al., 2012), pancreas and lung, kidney (Ge et al., 2005) in mice and humans, and slightly in brain, heart and hypophysis in humans (Klopper et al., 2008; Lu et al., 2010). High expression of bovine ANGPTL4 was found in adipose tissues and liver. Its expression was also detected in gastrointestinal tract (Mamedova et al., 2010), heart, kidney, hypophysis and skeletal muscle (Mamedova et al., 2010) of cattle. However, no study on expression of ovine ANGPTL4 gene has been reported.

Sheep tails fall into three types: long and short fat-tailed, and lean-tailed. There are significant differences in fat deposition in each type. Our early studies showed that mRNAs of UCP1 (Yuan et al., 2012), leptin (Lep) and its receptor (LEPR) genes (Liu et al., 2013) expressed differentially and spatiotemporally in two breeds with distinct tail types: Guangling Large Tailed (GLT) and Small Tailed Han (STH) sheep. In view of structure differences between species and the essential role in fat metabolism of ANGPTL4 gene, study on the cDNA sequence and expression of ovine ANGPTL4 gene may help us to understand the genetic mechanism of fat metabolism in different sheep breeds. The purpose of this study was to detect the mRNA expression in adipose tissues in the two breeds of GLT and STH based on the full coding sequence of ovine ANGPTL4 submitted to NCBI (GenBank accession number: KF8736131.1) by our research team.

\section{MATERIALS AND METHODS}

\section{Tissue sample preparation}

Adipose tissues were collected from GLT (Datong,
Shanxi, China) and STH (Jining, Shandong, China) after slaughtered at 2, 4, 6, 8, 10, 12 months of age, respectively, with four males and four females at each time point for both breeds. The tissues included great and small omental, subcutaneous, retroperitoneal, perirenal, mesenteric and tail fats. All samples were collected within 45 min postmortem, immediately frozen in liquid nitrogen, and subsequently stored at $-80^{\circ} \mathrm{C}$ until analysis. The feeding, management and slaughtering were conducted according to the National (GB 13078-2001and GB/T 17237-1998) and the Agricultural Standards (NY 5148-2002-NY 5151-2002) of the People's Republic of China. For details and the breed differences see Yuan (2012).

\section{Total RNA extraction and cDNA synthesis}

Total RNA from each sample was extracted via Trizol Reagent kit (Invitrogen, Carlsbad, CA, USA) according to the instruction of the manufacturer and detected by $1 \%$ agarose gel electrophoresis. The ratio of the absorbance at 260 and $280 \mathrm{~nm}(\mathrm{~A} 260 / 280)$ was used to assess the purity of nucleic acids (for pure RNA A260/280 is 1.8 2.0).

A Prime Script RT reagent kit (Takara Bio Inc, Takara code: DRR036A, Otsu, Shiga, Japan) was used to synthesize single-stranded cDNA from total RNA. The RT reaction was performed in $10 \mu \mathrm{L}$ of reaction mixture with $0.5 \mu \mathrm{g}$ total RNA. Reverse transcription was performed on a thermal cycler (Bio-Rad, Richmond, CA, USA) by using the following protocol: $37^{\circ} \mathrm{C}$ for $15 \mathrm{~min}$, and terminated by heating at $85^{\circ} \mathrm{C}$ for $5 \mathrm{~s}$ and cooling at $4^{\circ} \mathrm{C}$.

\section{Primer design}

Quantitative real-time polymerase chain reaction ( $Q R T$ $P C R)$ : primers were designed to span an exon-exon boundary based on the coding sequence of ovine ANGPTL4 gene. Ribosomal protein L 13 (RPL13) gene was chosen as house-keeping gene whose primers were designed based on ovine RPL13. All primers were designed by primer 3 plus (http://www.Biowebdb. org/primer3plus/). Primer sequence, PCR product size and annealing temperature are listed in Table 1.

Quantitative real-time PCR: Relative ANGPTL4 expression was quantified by using the $2^{-\Delta \Delta \mathrm{Ct}}$ method with an ABI Prism 7500 sequence detector (Applied Biosystems) and SYBR Premix Ex TaqTM kit (Takara, Japan), following the manufacturer's instructions. The qRT-PCR procedure was 45 cycles at $95^{\circ} \mathrm{C}$ for $30 \mathrm{~s}, 95^{\circ} \mathrm{C}$ for $5 \mathrm{~s}$ and $60^{\circ} \mathrm{C}$ for

Table 1. Primers used in qRT-PCR

\begin{tabular}{llccc}
\hline Gene & \multicolumn{1}{c}{ Primer sequence } & Length $(\mathrm{bp})$ & Annealing temperature $\left({ }^{\circ} \mathrm{C}\right)$ & Usage \\
\hline ANGPTL4 & QForward: ATTCCCCACCTTTCCTGGTT & 124 & 60 & mRNA \\
& QReverse: TCCGAAGCCATCTTTGTAGG & & & expression \\
\multirow{2}{*}{ RPL13 } & QForward: CTCAAGGTTGTGCGTCTGAA & 141 & 60 & \\
& QReverse: TTTCCGGTAGTGGATCTTGG & & & \\
\hline
\end{tabular}

qRT-PCR, quantitative real-time polymerase chain reaction; ANGPTL4, angiopoietin-like protein 4; RPL13, ribosomal protein L 13. 
34 s. Dissociation curves were obtained with following procedures: $95^{\circ} \mathrm{C}$ for $10 \mathrm{~s}, 60^{\circ} \mathrm{C}$ for $1 \mathrm{~min}$, then warming up from $60^{\circ} \mathrm{C}$ to $95^{\circ} \mathrm{C}$ slowly at the speed of $0.5^{\circ} \mathrm{C} / 10 \mathrm{~s}$. All samples and controls were run in tripartite, and the average of tripartite threshold cycle $(\mathrm{Ct})$ values was used in statistical analyses. The $\mathrm{Ct}$ values for all samples were normalized against that of RPL13 (Gebhardt et al., 2010).

\section{Statistical analysis}

General linear model was fitted and univariate analysis of variance (ANOVA) was conducted by SPSS17.0 (SPSS Inc., Chicago, IL, USA) to analyze factors influencing the mRNA expression. Breed, gender, tissue and age were included in the following model as the main factors:

$$
\begin{aligned}
y_{i j k l m}= & \mu+B i+G_{j}+T_{k}+M_{l}+B G_{i j}+B T_{i k}+B M_{i l} \\
& +G T_{j k}+G M_{j l}+T M_{k l}+e_{i j k l m}
\end{aligned}
$$

where, $y_{i j k l m}$ is the mRNA abundance; $\mu$ the overall mean; $B_{i}$ the $i_{t h}$ breed effect $(i=1,2) ; G_{j}$ the $j_{t h}$ gender effect $(j=1,2) ; T_{k}$ the $k_{t h}$ tissue effect $(k=1,2, \ldots, 7) ; M_{l}$ the $l_{t h}$ months of age effect $(l=2,4,6,8,10,12) ; B G_{i j}, B T_{i k}, B M_{i l}$, $G T_{j k}, G M_{j l}$, and $T M_{k l}$ the relevant two-way interactions; $e_{i j k l m}$ the random residue. Data expressed as $\bar{X}+G_{\bar{X}}$. Multiple comparisons among levels within factors were performed using the Duncan's method.

To characterize the temporal expression features of ANGPTL4 mRNA in the two breeds under study, the relative expressions in each and whole adipose tissue were also analyzed by fitting time-series models and conducted by Minitab 16 (Minitab Inc., State College, PA, USA). For clarifying the possible spatial expression features, Pearson correlation coefficients between expressions in seven depots were computed by using SPSS 17.0 software package.

\section{RESULTS}

\section{Factors influencing ovine ANGPTL4 mRNA expression}

ANOVA results showed that breed $(p<0.001)$, month of age $(p<0.001)$ and tissue $(p<0.001)$ were the main effects influencing the ANGPTL4 mRNA expression (Table 2). No significant effect was found for gender. Interaction between breed and month of age had highly significant $(\mathrm{p}<0.001)$ effect on the mRNA expression. Significant interactions $(p<0.05)$ were also found for breed $\times$ tissue and gender $\times$ tissue (Table 2).

\section{Differential mRNA expression within the main factors}

Table 3 gives the abundance of ANGPTL4 mRNA within the main factors. Abundance in GLT (4.566) was significantly higher than that in STH (1.753) suggesting that breed difference existed in the mRNA expression. Though there was no significant difference, slightly higher expression in females (3.229) than in males (3.077) was observed.

The highest mRNA expression was in subcutaneous (4.209) and tail (4.003) fats and the lowest expression in mesenteric fat $(1.156)$ with significant differences $(p<0.05)$ from those found in other adipose tissues. Moderate mRNA expression was found in great (2.457) and small omental (3.217), retroperitoneal (3.576) and perirenal (3.499) fats. These results suggested that the ovine ANGPTL4 gene expressed more in shallow than in deep fat depots.

The mRNA expression increased with month of age and reached the maximum at 10 months (4.797), and then decreased slightly at 12 months. Abundance at 10 months

\begin{tabular}{|c|c|c|c|c|}
\hline Source of variation & Sum of squares & Degree of freedom & $F$-value & Significance \\
\hline Model & $3,355.259$ & 65 & 6.292 & $* * *$ \\
\hline Intercept & $5,368.531$ & 1 & 654.182 & $* * *$ \\
\hline Breed & 797.801 & 1 & 97.216 & $* * *$ \\
\hline Gender & 2.916 & 1 & 0.355 & NS \\
\hline Month of age (MOA) & 408.555 & 5 & 9.957 & $* * *$ \\
\hline Tissue & 512.817 & 6 & 10.415 & $* * *$ \\
\hline Breed $\times$ MOA & 491.842 & 5 & 11.987 & $* * *$ \\
\hline Gender $\times$ MOA & 74.654 & 4 & 2.274 & NS \\
\hline Tissue $\times \mathrm{MOA}$ & 301.241 & 30 & 1.224 & NS \\
\hline Breed $\times$ gender & 1.471 & 1 & 0.179 & NS \\
\hline Breed×tissue & 113.715 & 6 & 2.309 & $*$ \\
\hline Gender $\times$ tissue & 106.109 & 6 & 2.155 & * \\
\hline Error & $4,390.469$ & 535 & & \\
\hline Total & $13,740.535$ & 601 & & \\
\hline Corrected total & $7,746.728$ & 600 & & \\
\hline
\end{tabular}

Table 2. Analysis of variance for the mRNA abundance of ovine ANGPTL4

ANGPTL4, angiopoietin-like protein 4; NS, not significant.

$* \mathrm{p}<0.05, * * \mathrm{p}<0.01, * * * \mathrm{p}<0.001$. 
Table 3. Abundance of ovine ANGPTL4 mRNA within the main factors

\begin{tabular}{|c|c|c|c|c|c|}
\hline Factor & Level & Abundance of mRNA* & Factor & Level & Abundance of mRNA* \\
\hline \multirow[t]{2}{*}{ Breed } & Small Tailed Han (STH) & $1.753 \pm 0.166^{\mathrm{b}}$ & Gender & Male & $3.077 \pm 0.169^{\mathrm{a}}$ \\
\hline & Guangling Large Tailed (GLT) & $4.566 \pm 0.170^{\mathrm{a}}$ & & Female & $3.229 \pm 0.167^{\mathrm{a}}$ \\
\hline \multirow[t]{7}{*}{ Adipose tissue } & Great omental fat (GO) & $2.457 \pm 0.311^{\mathrm{b}}$ & Month of age & 2 & $2.402 \pm 0.263^{\mathrm{b}}$ \\
\hline & Small omental fat (SO)) & $3.217 \pm 0.311^{\mathrm{b}}$ & & 4 & $2.549 \pm 0.281^{\mathrm{b}}$ \\
\hline & Subcutaneous fat (SC) & $4.209 \pm 0.311^{\mathrm{a}}$ & & 6 & $2.911 \pm 0.273^{\mathrm{b}}$ \\
\hline & Retroperitoneal fat (RP) & $3.576 \pm 0.313^{\mathrm{b}}$ & & 8 & $3.088 \pm 0.272^{\mathrm{b}}$ \\
\hline & Perirenal fat (PR) & $3.499 \pm 0.311^{\mathrm{b}}$ & & 10 & $4.797 \pm 0.273^{\mathrm{a}}$ \\
\hline & Mesenteric fat (MT) & $1.156 \pm 0.311^{\mathrm{bc}}$ & & 12 & $3.260 \pm 0.469^{\mathrm{b}}$ \\
\hline & Tail fat (TA) & $4.003 \pm 0.311^{\mathrm{a}}$ & & & \\
\hline
\end{tabular}

ANGPTL4, angiopoietin-like protein 4.

* Values with different superscripts within the same factor differ significantly $(\mathrm{p}<0.05)$.

was significantly different from those at other five age points, among which there was no significant difference.

\section{Differential mRNA expression between the main factors}

Besides the overall higher mRNA expressions in GLT than in STH, the expression trend in GLT was basically consistent with the general trend for main factor of months of age except slightly lower expression at eight months. However, higher mRNA expressions at 2 and 12 months were found in STH (Figrue 1A). The differences resulted in the significant interaction $(\mathrm{p}<0.001)$ between breed and month of age.

The significant interaction between gender and tissue $(p<0.05)$ can be attributed to the higher mRNA expression in retroperitoneal and lower expressions in perirenal and tail fats in males than females (Figure 1B).

Due to the higher mRNA expression in retroperitoneal and lower expression in perirenal fats in STH than GLT (Figure 1C), significant breed by tissue interaction was observed.

\section{The temporal expression features}

Figure 2 lists the observed and predicted relative expressions of ANGPTL4 mRNA in adipose tissues of Small Tailed Han sheep. In all the adipose tissues as a whole, the mRNA expressions from four to eight months of age were lower than that during ten to two months of age. As a result, the mRNA expressed as an inverted parabolic curve for both the observed and predicted (Figure 2A). Similar quadratic curves were fitted to the expressions in small omental (Figure 2C), retroperitoneal (Figure 2D), mesenteric (Figure 2F) and tail fat (Figure 2G). Different from these expression patterns, an approximately linear decrease function for expression in great omental (Figure 2B) and linear increase functions in perirenal (Figure 2E) and subcutaneous fat (Figure $2 \mathrm{H}$ ) were fitted. These results suggested that the mRNA expressed temporally and spatially in adipose tissues of STH in different manner, although the goodness of fit was not high.
Figure 3 gives the observed and predicted relative expressions of ANGPTL4 mRNA in adipose tissues of Guangling Large Tailed sheep. As a whole, the mRNA expression increased from two month of age, reached the highest at 10 and then decreased rapidly at 12 month of age. Opposite to the trend in STH, the mRNA expressed as a parabolic curve for both the observed and predicted (Figure 3A). Similar linear increase functions were fitted to the expressions in great (Figure 3B) and small omental (Figure 3C), mesenteric (Figure 3F), and subcutaneous (Figure 3H) adipose tissues. Quadratic expressions were found in retroperitoneal (Figure 3D), perirenal (Figure 3E) and tail fat (Figure 3G). These results also revealed the tempospatial differences in mRNA expression in GLT.

Significant breed differences can be reflected by comparing Figure 2 with Figure 3. Opposite expression trends between breeds were found in great omental (linear decrease in STH vs linear increase in GLT), retroperitoneal (inverted parabolic expression in STH vs parabolic expression in GLT) and tail fat (nonlinear increase in STH vs nonlinear decrease in GLT). Similar trends but in a different manner were found in small omental and mesenteric (nonlinear increase in STH vs linear increase in GLT), and perirenal (linear increase in STH vs nonlinear increase in GLT) adipose tissues. Though linear increase functions were fitted for expression in subcutaneous fat from both breeds, the observed expression increased in STH from 10 to 12 months of age, but decreased in GLT. Furthermore, no matter which tissue and what manner, ANGPTL4 mRNA expressed increasingly from 10 to 12 months of age in STH, but decreasingly in GLT.

\section{The spatial expression features}

Table 4 presents the correlations among ANGPTL4 mRNA abundances in seven adipose tissues under study, aiming to examine the possible spatial expression associations. Generally, more strong positive correlations were found in GLT than that in STH. Specifically, in GLT, the expression in tail fat correlated with that in great 


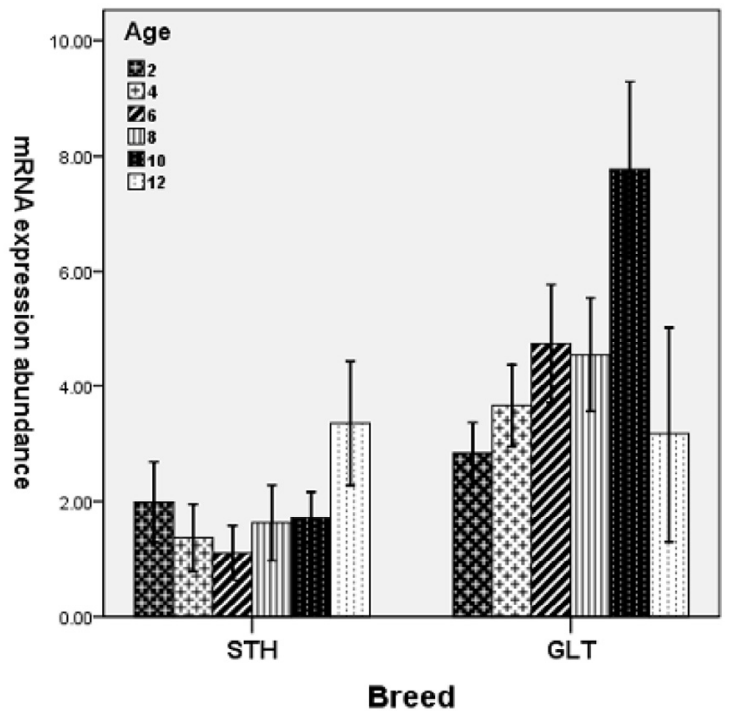

(A)

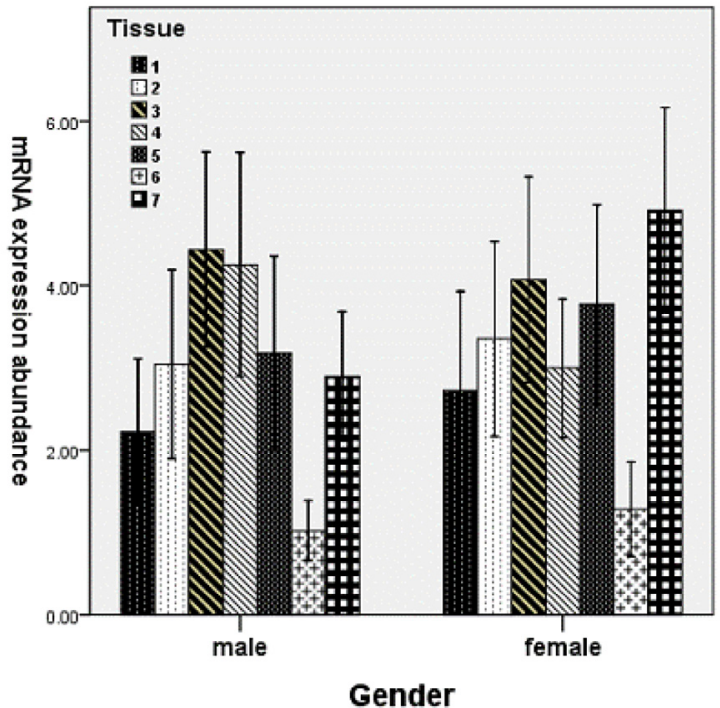

(B)

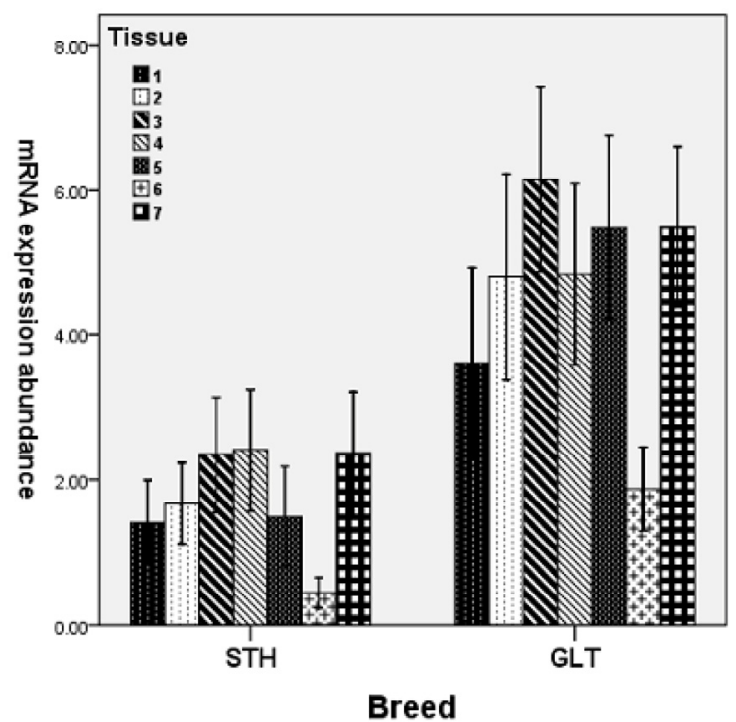

(C)

Figure 1. Effects of two-way interactions on the mRNA expression of ovine ANGPTL4 gene. (A) Breed $\times$ months of age, (B) Gender×tissue, (C) Breed×tissue. Breed: STH, Small Tailed Han sheep; GLT, Guangling Large Tailed sheep. Tissue: 1, great omental (GO); 2, small omental (SO); 3, subcutaneous (SC); 4, retroperitoneal (RP); 5, perirenal (PR); 6, mesenteric (MT); 7, Tail (TA).

omental (0.421), perirenal (0.469) and mesenteric (0.403) adipose tissues at highly significant level $(\mathrm{p}<0.01)$, respectively. Significant correlations were also found between expression in subcutaneous fat and that in great omental $(0.520, \mathrm{p}<0.01)$ and in retroperitoneal $(0.395$, $\mathrm{p}<0.05)$ fats. These results suggested that there seemed to be some kind of connection between the mRNA expressions in shallow and deep depots. Only one significant correlation was obtained between expressions in deep depots $(0.438$, between expressions in retroperitoneal and small omental adipose tissues).

In $\mathrm{STH}$, the mRNA expression in tail fat significantly correlated with that in small omental $(0.437, \mathrm{p}<0.01)$ and that in subcutaneous $(0.310, \mathrm{p}<0.05)$ adipose tissues, respectively. No significant correlation was found between deep depots for both breeds.

\section{DISCUSSION}

\section{Expression of ANGPTL4 mRNA}

Early studies on the mRNA expression of ANGPTL4 in both humans (Klopper et al., 2008) and rodents (Ge et al., 2005) suggested that the mRNA was highly expressed in adipose tissues and liver. In cattle, ANGPTL4 mRNA 


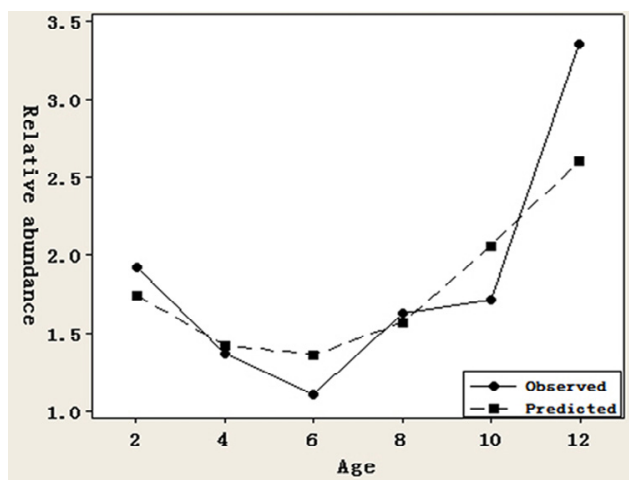

(A)

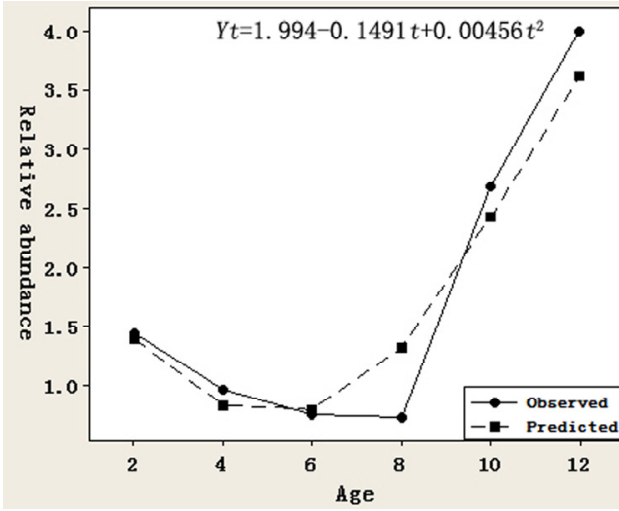

(C)

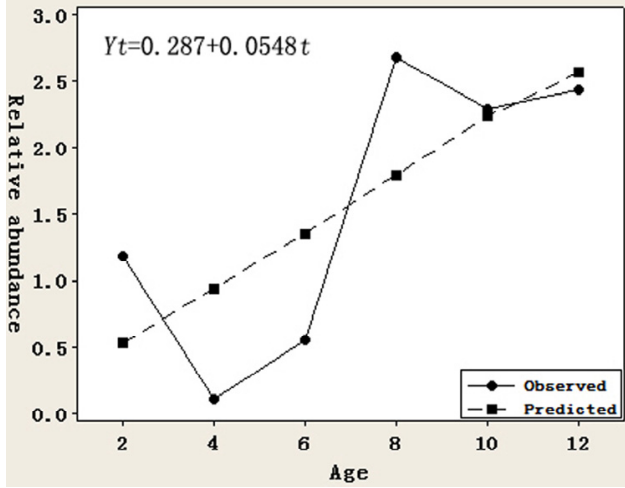

(E)

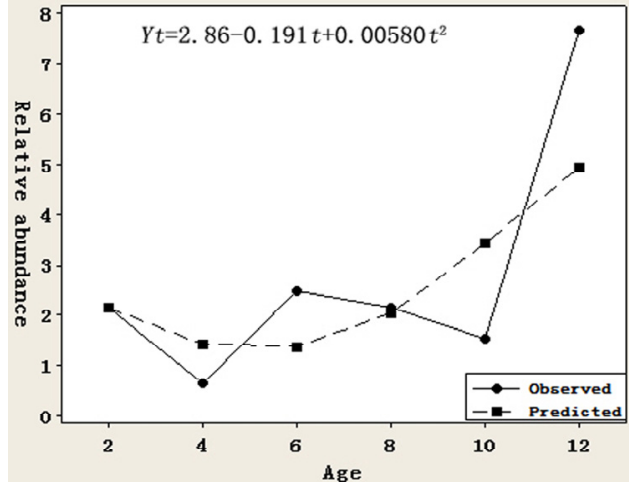

(G)

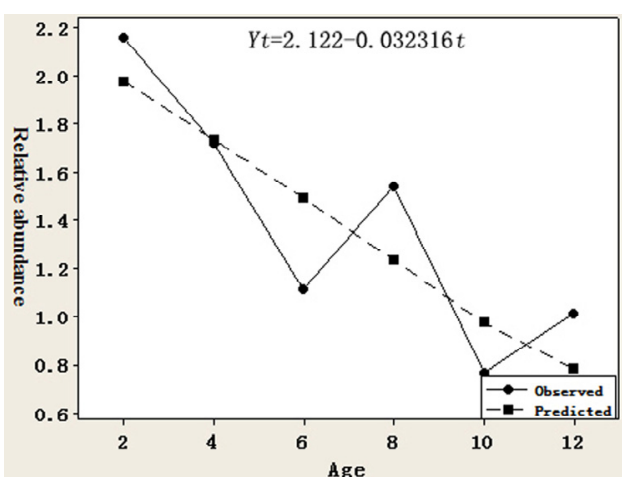

(B)

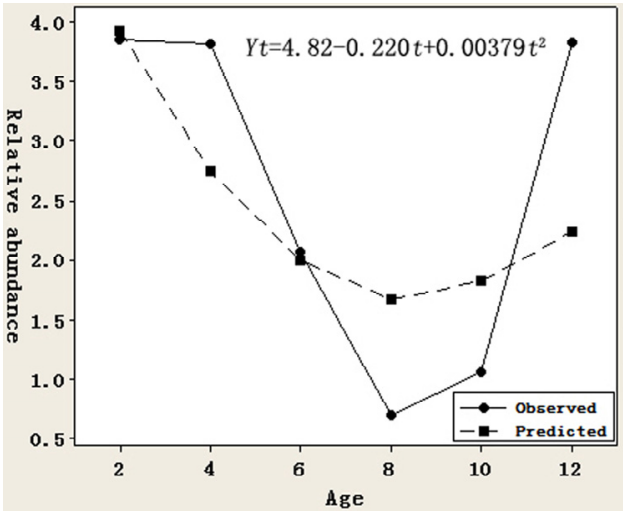

(D)

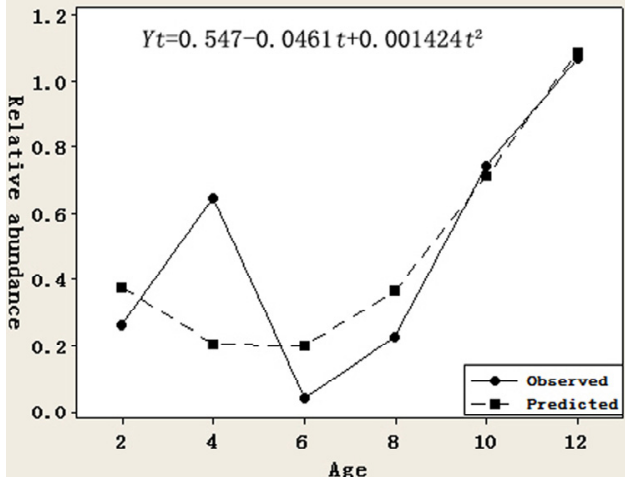

(F)

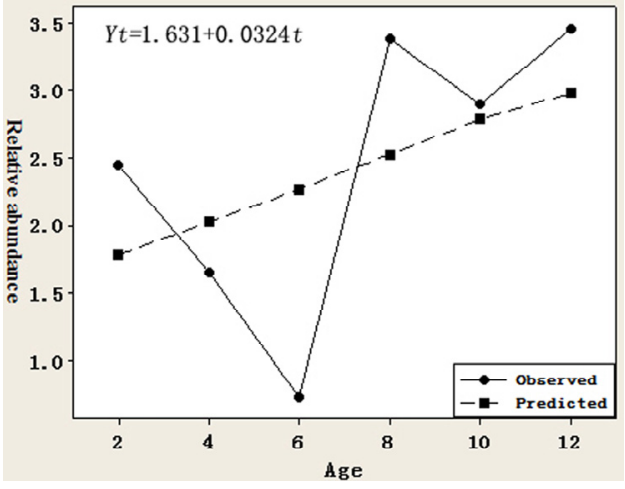

(H)

Figure 2. Observed (solid line) and predicted (dashed line) relative expressions of angiopoietin-like protein 4 (ANGPTL4) mRNA in adipose tissues of Small Tailed Han sheep. (A) whole adipose tissues, (B) great omental, (C) small omental, (D) retroperitoneal, (E) perirenal, $(\mathrm{F})$ mesenteric, $(\mathrm{G})$ tail fat, $(\mathrm{H})$ subcutaneous. 


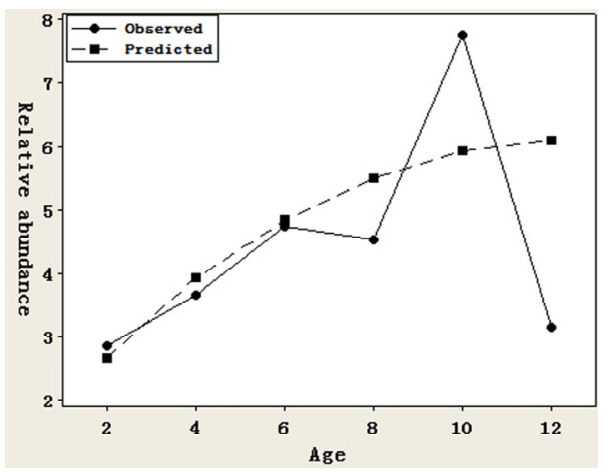

(A)

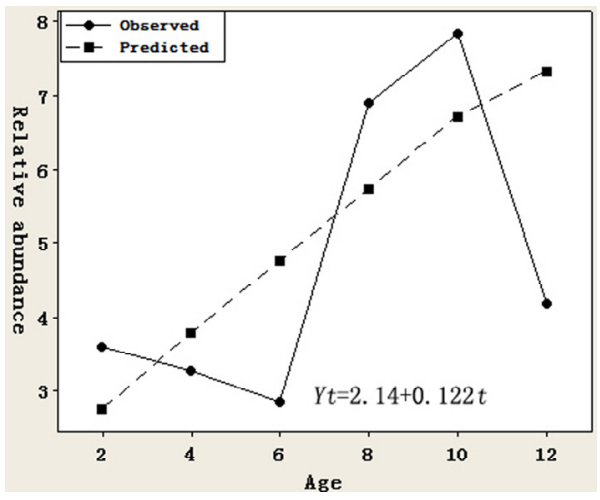

(C)

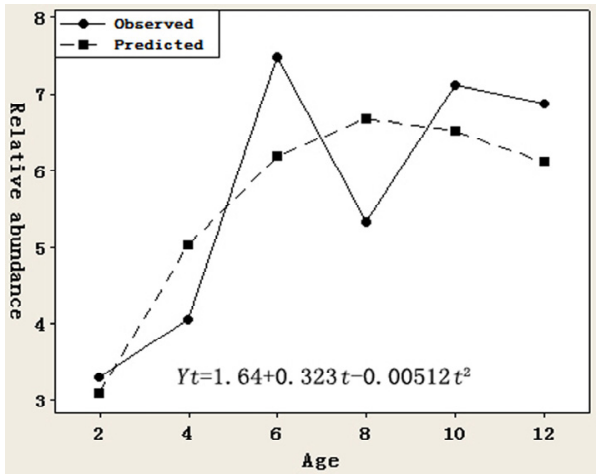

(E)

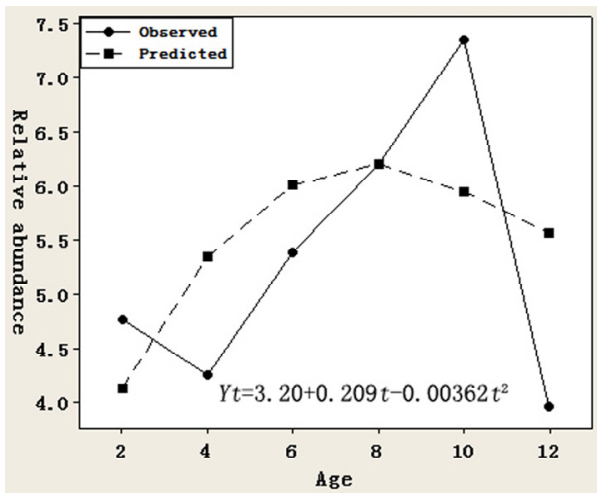

(G)

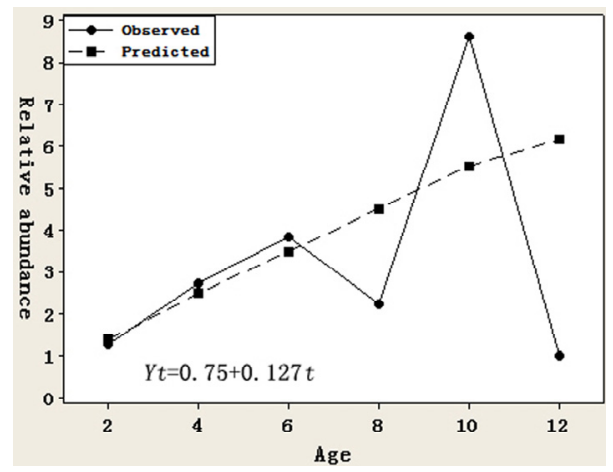

(B)

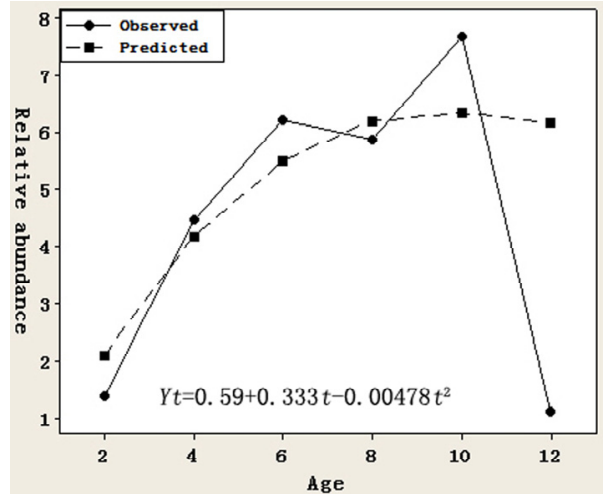

(D)

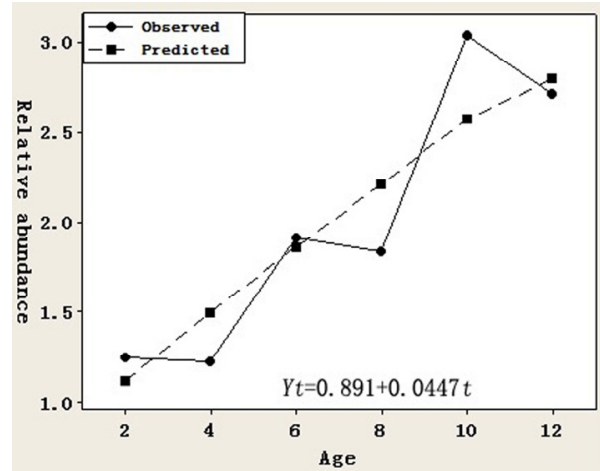

(F)

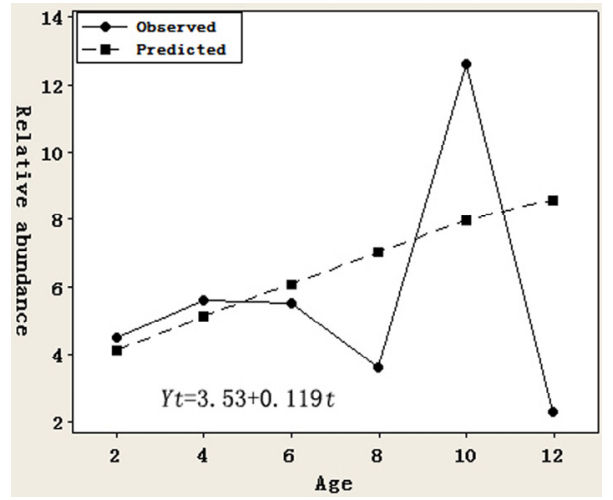

(H)

Figure 3. Observed (solid line) and predicted (dashed line) relative expressions of angiopoietin-like protein 4 (ANGPTL4) mRNA in adipose tissues of Guangling Large Tailed sheep. (A) whole adipose tissues, (B) great omental, (C) small omental, (D) retroperitoneal, (E) perirenal, $(\mathrm{F})$ mesenteric, $(\mathrm{G})$ tail fat, $(\mathrm{H})$ subcutaneous. 
Table 4. Spatial correlation between expressions of adipose tissues in STH (upper triangle) and GLT (lower triangle)

\begin{tabular}{llcccccc}
\hline & \multicolumn{9}{c}{ ANGPTL4 mRNA abundance } \\
\cline { 2 - 8 } & GO & SO & RE & PE & ME & TA & SU \\
\hline GO & & -0.085 & 0.259 & 0.096 & -0.143 & -0.161 & -0.007 \\
SO & 0.201 & & 0.070 & 0.129 & 0.204 & $0.437^{* *}$ & 0.008 \\
RE & 0.140 & $0.438^{* *}$ & & -0.259 & 0.149 & 0.024 & 0.181 \\
PE & 0.251 & -0.090 & 0.014 & & 0.282 & 0.232 & 0.140 \\
ME & 0.299 & 0.086 & -0.050 & 0.134 & & 0.025 & -0.122 \\
TA & $0.421^{* *}$ & -0.100 & -0.024 & $0.469^{* *}$ & $0.403 * *$ & $0.310^{*}$ \\
SU & $0.520^{* *}$ & 0.261 & $0.395^{*}$ & -0.015 & 0.245 & 0.131 & \\
\hline STH, & & & & & & \\
\end{tabular}

STH, Small Tailed Han; GLT, Guangling Large Tailed; ANGPTL4, angiopoietin-like protein 4; GO, great omental; SO, small omental; RE, retroperitoneal; PE, perirenal; ME, mesenterium; TA, tail fat; $\mathrm{SU}$, subcutaneous.

${ }^{* *} \mathrm{p}<0.01,{ }^{*} \mathrm{p}<0.05$.

abundance was also found substantially higher in adipose than in other tissues (Mamedova et al., 2010). For adipose tissue itself, the mRNA expression varied in different fat depots. In human, the ANGPTL4 expressed in visceral (omental) and had no expression in subcutaneous and breast (mammary) adipose tissues by immunohistochemical staining (Gentil et al., 2006). In mice, Dutton and Trayhurn (2008) found that both the mRNA and protein expressed in five adipose tissues, with expression in subcutaneous nonsignificantly lower than that in epididymal and perirenal depots. In this study, we detected mRNA expression signals in all seven tested adipose tissues, with the highest in subcutaneous followed by tail fat and the least in mesenteric adipose tissue. Our results indicated that ovine ANGPTL4 expressed higher in shallow than in visceral adipose depots, which seems to be opposite to the results obtained in both humans and mice. This divergence may due to species difference, and more possibly to the directionally artificial selection for meat purpose in sheep.

Breed difference exists in ovine ANGPTL4 mRNA expression with higher expression in GLT than in STH. GLT, one type of long fat-tailed breed, has high meat quality, strong ability of lipid accumulation and weak fertility. STH, one type of short fat-tailed breed, however, has high fertility with precocious trait but weak lipid accumulation (Yuan et al., 2012). ANGPTL4 can cause accumulation of TG by inhibiting activity of LPL, and thus leads to strong ability of lipid accumulation in GLT. Our result was supported by the findings that ANGPTL4 expression in adipose tissue from obese pig breeds was significantly higher than that from lean pig breeds (Feng et al., 2006).

Nutritional condition and seasonal temperatures can affect the expression of ANGPTL4 mRNA. In this study, ages at 2, 4, 6, 8, 10, and 12 months corresponds to Jan, Mar, May, Jul, Sep and Nov, respectively. Two flocks were raised indoors before weaning and during dry seasons in winter and spring seasons from Dec to May, and by grazing on pasture in grass seasons from Jun to Nov (Yuan et al., 2012). The highest and lowest mRNA expressions in adipose tissues were detected in Sep and Jan, respectively. This differential expression depends on the supply of feed and accumulation of fat. When sufficient feed is supplied and animals have good body condition in grass seasons, especially for animals at 10 months of age, the mRNA expressed at higher levels than that in dry seasons. This result was supported by studies, e.g. in mice. Camenisch (2002) reported that the expression level of ANGPTL4 rose clearly when mice had high fat diet.

In view of the complex factors, especially the age effect may be confused by season effect in naturally managed sheep flock. Though time-series analysis was used intensively in gene expression studies, most for microarray gene expression data, the experimental subjects were wellcontrolled rather than natural, e.g. Wong et al. (2015) and Yang et al. (2012). Moreover, model fitting considered the influence of time on the ANGPTL4 mRNA expression only, ignoring the influence of other factors on the gene expression. So the results by time-series analysis are not quite consistent with those obtained by qRT-PCR. This seems to suggest that time-series gene expression analysis is more suitable for the well-controlled experimental design.

More strong positive correlations between ANGPTL4 mRNA expressions in shallow and in deep adipose tissues were found in GLT than that in STH by spatial correlation analysis. These results further indicated the breed differences. However, why there was no significant association between deep depots for both breeds is difficult to understand and deserves further study.

\section{CONCLUSION}

Ontogenetic mRNA expression of ANGPTL4 in seven adipose tissues from two different sheep breeds was studied by quantitative real time PCR and analyzed by ANOVA and time series analysis. The ANGPTL4 mRNA expresses spatiotemporally and differentially in adipose tissues of both breeds. Breed, month of age and adipose tissues from different depots influence the mRNA expression 
significantly. The season effect on the mRNA expression can to a certain degree confuse the age effect for naturally managed sheep flocks.

\section{CONFLICT OF INTEREST}

We certify that there is no conflict of interest with any financial organization regarding the material discussed in the manuscript.

\section{ACKNOWLEDGMENTS}

The authors thank the staff in the Department of Animal Genetics, Breeding and Reproduction, Shanxi Agricultural University for providing the research laboratories. We thank the guidance of Li Baojun in manuscript preparation. This work was supported by the project of the Chinese National Natural Science Foundation (No. 30972084), the project of Young Academic Leaders of Shanxi Agricultural University (No. XD201205), and the Key Scientific and Technological Projects of Shanxi Province (011029).

\section{REFERENCES}

Camenisch, G., M. T. Pisabarro, D. Sherman, J. Kowalski, M. Nagel, P. Hass, M. H. Xie, A. Gurney, S. Bodary, X. H. Liang, K. Clark, M. Beresini, N. Ferrara, and H. P. Gerber. 2002. ANGPTL3 stimulates endothelial cell adhesion and migration via integrin av $\beta 3$ and induces blood veddel formation in vivo. J. Biol. Chem. 277:17281-17290.

Dijk, W. and S. Kersten. 2014. Regulation of lipoprotein lipase by Angptl4. Trends Endocrinol. Metab. 25:146-155.

Dutton, S. and P. Trayhurn. 2008. Regulation of angiopoietin-like protein 4/fasting-induced adipose factor (Angpt14/FIAF) expression in mouse white adipose tissue and 3T3-L1 adipocytes. Br. J. Nutr. 100:18-26.

Feng, S. Q., X. D. Chen, T. Xia, L. Gan, H. Qiu, M. H. Dai, L. Zhou, Y. Peng, and Z. Q. Yang. 2006. Cloning, chromosome mapping and expression characteristics of porcine ANGPTL3 and -4. Cytogenet. Genome Res. 114:44-49.

Feingold, K. R., J. K. Shigenaga, A. S. Cross, A. Moser, and C. Grunfeld. 2012. Angiopoietin like protein 4 expression is decreased in activated macrophages. Biochem. Biophys. Res. Commun. 421:612-615.

Ge, H., J. Y. Cha, H. Gopal, C. Harp, X. Yu, J. J. Repa, and C. Li. 2005. Differential regulation and properties of angiopoietinlike proteins 3 and 4. J. Lipid Res. 46:1484-1490.

Gebhardt, F. M., H. A. Scott, and P. R. Dodd. 2010. Housekeepers for accurate transcript expression analysis in Alzheimer's disease autopsy brain tissue. Alzheimers Dement. 6:465-474.

Gentil, C., S. Le Jan, J. Philippe, J. Leibowith, P. Sonigo, S. Germain, and F. Piétri-Rouxel. 2006. Is oxygen a key factor in the lipodystrophy phenotype? Lipids Health Dis. 5:27.
Georgiadi, A. and S. Kersten. 2012. Mechanisms of gene regulation by fatty acids. Adv. Nutr. 3:127-134.

Gray, N. E., L. N. Lam, K. Yang, A. Y. Zhou, S. Koliwad, and J. C. Wang. 2012. Angiopoietin-like 4 (Angpt14) protein is a physiological mediator of intracellular lipolysis in murine adipocytes. J. Biol. Chem. 287:8444- 8456.

Grootaert, C., T. Van de Wiele, W. Verstraete, M. Bracke, and B. Vanhoecke. 2012. Angiopoietin-like protein 4: health effects, modulating agents and structure-function relationships. Expert. Rev. Proteomics 9:181-199.

Kadomatsu, T., M. Tabata, and Y. Oike. 2011. Angiopoietin-like proteins: emerging targets for treatment of obesity and related metabolic diseases. FEBS J. 278:559-564.

Klopper, J. P., A. Berenz, W. R. Hays, V. Sharma, U. Pugazhenthi, J. Janssen, M. Singh, R. P. Bissonnette, and B. R. Haugen. 2008. In vivo and microarray analysis of rexinoid-responsive anaplastic thyroid carcinoma. Clin. Cancer Res. 14:589-596.

Liu, B. F., S. S. Zhou, J. L. Wang, L. L. Wei, L. Y. Qiao, J. H. Liu, C. Liang, and W. Z. Liu. 2013. Differential expression of Lep and LEPR mRNA in adipose tissues of sheep with divergent fat-tails[in Chinese]. Acta Veterinaria et Zootechnica Sinica 44:1014-1022.

Lu, B., A. Moser, J. K. Shigenaga, C. Grunfeld, and K. R. Feingold. 2010. The acute phase response stimulates the expression of angiopoietin like protein 4. Biochem. Biophys. Res. Commun. 391:1737-1741.

Mandard, S., F. Zandbergen, N. S. Tan, P. Escher, D. Patsouris, W. Koenig, R. Kleemann, A. Bakker, F. Veenman, W. Wahli, M. Müller, and S. Kersten. 2004. The direct peroxisome proliferator-activated receptor target fasting-induced adipose factor (FIAF/PGAR/ANGPTL4) is present in blood plasma as a truncated protein that is increased by fenofibrate treatment. J. Biol. Chem. 279:34411-34420.

Mandard, S., F. Zandbergen, E. W. Van Straten, W. Wahli, F. Kuipers, M. Müller, and S. Kersten. 2006. The fasting-induced adipose factor/angiopoietin-like protein 4 is physically associated with lipoproteins and governs plasma lipid levels and adiposity. J. Biol. Chem. 281:934-944.

Mamedova, L. K., K. Robbins, B. J. Johnson, and B. J. Bradford. 2010. Tissue expression of angiopoietin-like protein 4 in cattle. J. Anim. Sci. 88:124-130.

Wong, K., F. Sun, G. Trudel, P. Sebastiani, and O. Laneuville. 2015. Temporal gene expression profiling of the rat knee joint capsule during immobilization-induced joint contractures. BMC Musculoskelet. Disord. 16:125.

Yang, Q., J. S. Mattick, M. A. Orman, T. T. Nguyen, M. G. Ierapetritou, F. Berthiaume, and I. P. Androulakis. 2012. Dynamics of hepatic gene expression profile in a rat cecal ligation and puncture model. J. Surg. Res. 176:583-600.

Yuan, Y. N., W. Z. Liu, J. H. Liu, L. Y. Qiao, and J. L. Wu. 2012. Cloning and ontogenetic expression of the uncoupling protein 1 gene UCP1 in sheep. J. Appl. Genet. 53:203-212.

Zhu, P., Y. Y. Goh, H. F. Chin, S. Kersten, and N. S. Tan. 2012. Angiopoietin-like 4: A decade of research. Biosci. Rep. 32: 211-219. 